\title{
Modelling the Potential Acute and Post-Acute Burden of COVID-19 Under the Australian Border Re-Opening Plan
}

\author{
Mary Rose Angeles \\ Institute for Health Transformation, Faculty of Health, Deakin University \\ Sithara Wanni Arachchige Dona \\ Institute for Health Transformation, Faculty of Health, Deakin University \\ Dieu Nguyen \\ Institute for Health Transformation, Faculty of Health, Deakin University \\ Long Le \\ Institute for Health Transformation, Faculty of Health, Deakin University \\ Martin Hensher ( $\square$ martin.hensher@deakin.edu.au ) \\ Institute for Health Transformation, Faculty of Health, Deakin University
}

\section{Research Article}

Keywords: Burden of disease, Disability-adjusted life years, DALY, Coronavirus, Covid-19, Long COVID, post-COVID, Post-acute COVID, Australia

Posted Date: November 30th, 2021

DOI: https://doi.org/10.21203/rs.3.rs-1066181/v1

License: (c) (7) This work is licensed under a Creative Commons Attribution 4.0 International License. Read Full License

Version of Record: A version of this preprint was published at BMC Public Health on April 14th, 2022. See the published version at https://doi.org/10.1186/s12889-022-13169-x. 


\section{Abstract \\ Background}

Concerns have grown that post-acute sequelae of COVID-19 may affect significant numbers of survivors. However, the analyses used to guide policy-making for Australia's national and state re-opening plans have not incorporated non-acute illness in their modelling. We therefore develop a model by which to estimate the potential acute and post-acute COVID-19 burden using disability-adjusted life years (DALYs) associated with the re-opening of Australian borders and the easing of other public health measures, with particular attention to longer term, post-acute consequences and the potential impact of permanent functional impairment following COVID-19.

\section{Methods}

A model was developed to estimate the burden of COVID-19 using DALYs. It was then applied to different scenarios drawn from the Doherty Institute's modelling report, to estimate the likely DALY losses under the Australian national reopening plan. Uncertainty and sensitivity analysis were performed to examine the robustness of the results.

\section{Results}

Mortality was responsible for $72 \%-74 \%$ of the total base case COVID-19 burden. Long COVID and post-intensive care syndrome accounted for at least $19 \%$ and $3 \%$ of the total base case DALYs respectively. When included in the analysis, permanent impairment could contribute up to $51 \%-55 \%$ of total DALYs lost.

\section{Conclusions}

The impact of Long COVID and potential long-term post-COVID disabilities could contribute substantially to COVID-19 burden in Australia's post-vaccination setting. As vaccination coverage increases, the share of COVID-19 burden driven by longer-term morbidity rises relative to mortality. As Australia re-opens, better estimates of COVID-19 burden can assist with decision-making on pandemic control measures and planning for the healthcare needs of COVID-19 survivors. Our estimates highlight the importance of valuing the morbidity of post-COVID-19 sequelae, above and beyond simple mortality and case statistics.

\section{Background}

In response to the coronavirus disease 2019 (COVID-19) pandemic, Australian governments implemented a number of strict strategies, including international border closure, intermittent interstate border closure and lockdowns, effectively aiming to achieve COVID-19 zero [1]. Australia's first pandemic measure began on the 15th of March 2020, when international arrivals were mandated to self-quarantine for 14 days. The government also imposed social distance rules and restriction over non-essential gatherings three days after. The closure of non-essential business such as places of worships, gyms, recreational venues were also mandated on the 21st of March 2020 [2]. Tighter measures were implemented on the 30th of March 2020 where "stay at home" orders were imposed except for essential activities such as medical care, exercise, food shopping. International travel has been severely restricted, not only through strict arrival caps and quarantine requirements for incoming travellers, but also by federal restrictions on the ability of Australian citizens and residents to leave the country without special exemptions for travel. Travel between states has been significantly reduced due to periodic interstate border restrictions (and, indeed, restrictions on internal travel within some states). Since early 2020 , the precise public health measures in use have differed between each state and territory in Australia; restrictions have been periodically tightened and relaxed depending on levels of transmission, case numbers and perceived risk levels [2-4].

While these measures have been effective in reducing the number of COVID-19 cases and deaths compared to other nations, Australia has suffered significant economic losses as a result [1]. Vaccination is currently the central strategy for returning to pre-pandemic social lifestyles and economic activity. Although COVID-19 vaccines cannot fully contain the spread of transmission, they offer significant protection from more severe health impacts of COVID-19 [1]. The National Cabinet (comprising the federal Prime Minister and the Premier / First Ministers of each state and territory) has agreed a national reopening plan[5], under which Australia is now transitioning from pre-vaccination to post-vaccination settings, and moving towards a "living with COVID-19 strategy", re-opening its international borders, and relaxing restrictions and lockdowns.

To support the development of this plan, the Doherty Institute was commissioned by the National Cabinet to model the impact of different reopening scenarios, based on vaccination coverage, public health and social measures (PHSM), efficacy of test, trace, isolate, quarantine (TTIQ), with consideration of seeding infection growth [6]. The Doherty modelling provided detailed outputs on new cases, hospitalisation and mortality, but did not estimate the potential longer-term consequences of post-acute COVID-19 sequelae from these reopening strategies. 'Long COVID' is referred to as the extended and more complex course of COVID-19 as a multisystem disease associated with a range of symptoms [7]. In addition, there is increasing evidence of organ impairment and late complications in some COVID-19 survivors [7-9]. This raises concerns that some patients will experience long-term or permanent disability following COVID19; while historical evidence of long-term sequelae following viral pandemics also suggests we should be on our guard for the emergence of other long-term impacts in a small proportion of survivors [7].

To better understand the dynamics and relative impact of COVID-19, the aim of our model is to supplement the Doherty modelling by estimating the full burden of COVID-19 in Australia's border reopening strategy using disability adjusted life years (DALYs), paying particular attention to post-acute consequences and the potential impact of permanent functional impairment as a consequence of COVID-19. Readers should note that the authors of this 
paper were not involved in the Doherty Institute modelling exercise, and our modelling results should not be interpreted as bearing the endorsement of the Doherty Institute team.

\section{Methods}

A decision tree was developed based on Burden-eu's protocol guideline and consensus model to estimate the DALY loss from the modelled COVID-19 cases of Australia's national roadmap to re-opening $[6,10,11]$.

\section{Model structure}

The model in this study was developed in Microsoft Excel 2016 (see Additional file 1 for the DALY COVID-19 model) [10, 11]. Figure 1 presents the COVID-19 outcome model adapted in this study $[10,11]$. Patients infected with COVID-19 were simulated in the model and could progress to various health states: asymptomatic, symptomatic (acute), or death [10,11]. "Asymptomatic" refers when a patient contracted the virus but do not show any symptoms [10, 11]. In the symptomatic health state, acute cases may experience mild/moderate symptoms but are not hospitalised, severe symptoms with hospitalisation, or a critical condition managed in intensive care unit (ICU). Asymptomatic and symptomatic patients may experience full recovery, Long COVID, or a permanent functional impairment $[10,11]$. Long COVID patients experience longer-term symptoms after an acute COVID-19 infection, and may not recover fully for some time. Over time, those with Long COVID either fully recover or develop permanent functional impairment. Permanent functional impairments included diabetes, Parkinson's disease, dementia, anxiety disorders, and ischemic stroke. Patients managed in ICU may recover fully or develop post-intensive care syndrome (PICS). PICS is defined as "new or worsening impairments in physical, cognitive, or mental health status arising after critical illness and persisting beyond acute care hospitalisation" [12]. It is assumed that patients in the state of permanent functional impairment could not transition back to post-acute consequence and recovery state.

\section{Disability adjusted life years}

Disability-adjusted life years (DALYs) were estimated by summing the life years lost due to premature mortality (YLL) and the years lived with disability (YLD) primarily using an incidence-based approach $[10,11]$.

$D A L Y=Y L L+Y L D$

YLL is calculated from the COVID-19 mortality statistics (M) and average life expectancy (LE), presented into a 10-age group band generated from public national data sources $[6,13]$.

YLL=M $\times$ LE

YLD for acute COVID-19, Long COVID and PICS (YLDinc) was calculated by multiplying the number of COVID-19 cases (N), average duration of health state until recovery or death (D), and disability weight (DW). The disability weight accounts for the extent of health loss associated with the specific health outcomes which range from 0 to 1 ( $0=$ no impact or having full health, $1=$ occurrence of death) [14].

YLDinc=N $\times$ D x DW

Some COVID-19 survivors have developed permanent illness or disability (distinct from Long COVID) [7-9]. However, the evidence on permanent impairment is less robust than that for acute, Long COVID and PICS. We therefore illustrate separately some of the potential for long-term disability due to COVID-19.

Conservatively, we only include the incidence of certain permanent functional impairments post-COVID most commonly observed in large cohort studies (e.g., new onset of diabetes, Parkinson's disease, dementia, anxiety disorders, and ischemic stroke) [8, 9]. To estimate the DALYs associated with these conditions, we sourced DALYs per person with these conditions from the 2019 Australian Burden of Disease study (See Additional file 2 Table 4) [15].

The results of this model are presented in three scenarios. The base case DALYs consist of the mortality and morbidity impact of acute COVID-19, Long COVID and PICS. "Total Burden One" presents the base case result plus the potential impact of all listed permanent functional impairments. "Total Burden Two" excludes diabetes as a permanent impairment.

\section{Data Inputs}

The data were obtained from the literature and Doherty's modelling report[6], as update in September 2021. See Additional file 2 for more information. Ethics approval was not required as this study analysed publicly available data.

\section{Doherty COVID-19 modelling}

The Doherty COVID-19 model[6] was developed to inform Australia's national COVID-19 reopening plan; it estimated the potential health and health system impacts of COVID-19 after eligible Australians achieve different coverage levels of full doses of COVID-19 vaccines (i.e. 50-80\%). Transmission potential of COVID-19 delta variant, different bundles of public health and social distancing measures (PHSM), the efficacy of test-trace-isolate-quarantine activities (TTIQ), and the seeding infection rate ("initial number of daily cases present in the population at a given vaccination threshold") were included in the Doherty analysis. Doherty model outputs over the first 180 days were reported in much detail [6], enabling us to calculate the potential DALY burden for each strategy 
and allowing us to illustrate the likely DALY burden arising from the post-acute consequences of COVID-19. The results and the analysis of our model are generated based on the most applicable hypothetical Doherty scenarios, given actual developments towards reopening to date across Australia. All other scenarios were also presented and calculated in the additional file 1 and additional file 2 table 1.

- Scenario 2C: Outbreaks seeded with 1,000 to 4,500 cases given partially effective TTIQ. The community has achieved COVID-19 vaccination coverage of $70 \%$ while maintaining low PHSM.

- Scenario 2D: Outbreaks seeded with 1,000 to 4,500 cases given partially effective TTIQ. The "medium PHSMs are overlaid between the 70 and $80 \%$ coverage thresholds with reversion to low PHSMs thereafter".

- Scenario 3B: Outbreaks seeded with 300-1,000 cases given partially effective TTIQ. The community has achieved COVID-19 vaccination coverage of 80\% with baseline PHSM.

- Scenario 3C: Outbreaks seeded with 1,000 to 4,500 cases given partially effective TTIQ. The community has achieved COVID-19 vaccination coverage of $80 \%$ with baseline PHSM.

The full definition of TTIQ and different levels of PHSM are reported elsewhere [6, 16]. Briefly, due to the high volume of cases, expected delays in TTIQ responses are noted for "partial" TTIQ [6]. No "stay-at-home" orders, but low-density requirements (2 sqm rule) are imposed for "baseline" PHSM. Social distancing rules are still mandated, but retail trade and travel restrictions are not imposed [6, 16]. Rules mandated for baseline are also similar for "low" PHSM, however there are some limitations in recreational, retail and workplace capacity under "low" PHSM [6, 16]. Under medium PHSM, "stay-at-home" orders are imposed unless for work, study and essential activities. However, work from home is recommended when possible. Schools, childcare and indoor recreational venues are closed. Intra and interstate travel are not allowed under medium PHSM $[6,16]$.

\section{Asymptomatic Health State}

According to a systematic review and meta-analysis, approximately $17 \%$ (95\% Cl:14\%-20\%) of total cases are asymptomatic [17]. However, asymptomatic cases are not presented in Doherty's modelled result and for this reason we did not include asymptomatic cases in this calculation.

\section{Symptomatic Health State}

COVID-19 cases were obtained from the Doherty Modelling Interim Report to national Cabinet (17th September 2021) [6], and a period of 14-days was conservatively and collectively used for the recovery duration for acute-COVID-19 state [18].

\section{Post-acute Consequences}

Our model assumed that Long COVID symptoms start directly after the symptomatic phase. Given the United Kingdom (UK) evidence that some patients are still reporting Long COVID over 12 months after infection [19], our model assumed that Long COVID can potentially last up to two years. Due to the lack of longitudinal data regarding the length of Long COVID, we extrapolated available data on numbers of Long COVID cases over time from the UK Office of National Statistics (ONS)[19] and from a population-based cohort study in New South Wales(NSW)[20] until it reached 0\% using a fitted decay function [21]. This estimate was only applied to COVID-19 survivors.

According to a large case-control study in the UK, fully vaccinated individuals appear less likely to experience Long COVID following "breakthrough" infection compared with their unvaccinated counterparts (odds ratio $=0.51,95 \% \mathrm{Cl}: 0.32-0.82$ ) [22]. This OR was converted to relative risk using the Cochrane formula[23] and was then applied in the vaccinated cohort.

\section{Permanent functional impairment}

To quantify the incidence of permanent disability post COVID-19, we used data from two studies that investigated these conditions [8, 9]. A large US cohort study indicated the incidence of $0.11 \%(95 \% \mathrm{Cl}: 0.08-0.14)$ for Parkinson's disease, $0.67 \%$ (95\% Cl:0.59-0.75) for dementia, $7.11 \%(95 \% \mathrm{Cl}: 6.82-7.41)$ for anxiety disorders and $0.76 \%(95 \% \mathrm{Cl}: 0.68-0.85)$ for ischemic stroke within six months post-COVID-19 [9]. For diabetes, data from a large cohort study in the UK found that $2.83 \%$ (95\% Cl:2.57-3.12) of hospitalised patients were diagnosed with diabetes (type-1 or-2) over a mean follow-up of 140 days [8]. However, it is arguable whether diabetes was an undetected pre-existing condition or whether COVID-19 induced type-1 or type-2 diabetes [24, 25]. Thus, we presented our DALY calculation with ("Total Burden One") and without the impact of diabetes ("Total Burden Two").

\section{Post-Intensive care syndrome}

We also included the debilitating effects often seen after ICU admission, commonly referred to as post-intensive care syndrome (PICS) [26]. The only cohort study that investigated PICS in the COVID-19 population found that $90.60 \%$ of ICU survivors had PICS [26]. Unfortunately, there are no existing disability weights for PICS. A QALY utility score of 0.75 (95\% Cl: 0.63-0.84) was available for ICU COVID-19 survivors $12-16$ weeks post discharge [27], hence a comparable illness with similar utility score was assumed. We selected atrial fibrillation and flutter which has a utility score of $0.75[28]$ and a disability weight of 0.22 (95\% Cl: $0.15-0.31$ ) [29]. A lifetime duration was assumed for PICS reflecting study findings that followed ICU survivors over 5-years and 10-years [30, 31]. 


\section{Uncertainty Analysis and pilot model testing}

Uncertainty analyses were undertaken to propagate parameter uncertainty (i.e. sampling error) from the input parameters to the final model outputs. Monte Carlo simulation with 2,000 iterations via the add-in tool Ersatz (Ersatz, Version 1.35) was used [32]. Estimates of DALYs were presented with 95\% uncertainty intervals $(95 \% \mathrm{UI})$.

The pilot testing of the model was conducted using actual observed Australian COVID-19 cases, hospitalisations and deaths data from January 2020 to January 2021. In this pilot, we have conducted different sensitivity analysis on rates of acute health states, post-acute rates, and a 28-day recovery period from the acute health state, to explore whether these input parameters have impacted on the robustness of the results (See additional file 3 ). Findings showed only a very small change in DALYs. Therefore, we have not included these sensitivity analyses in the current study. In this study, we have conducted a sensitivity analysis to investigate the impact of changes in the rates for post-acute consequences, using NSW Long COVID data points starting at $34 \%$ at week three and $0 \%$ at week 104 [20].

\section{Results}

Tables 1-3 (See additional file 4 for full results and corresponding $95 \% \mathrm{Cls}$ ) show the distribution of health states contributing to the total morbidity and DALY burden using the ONS data points. In all scenarios, the mortality impact (YLL) is the largest contributor to the base case DALY burden (72\%-74\%), followed by the morbidity impact of Long COVID (19-22\%). However, including the potential for permanent functional impairment over a lifetime had a much bigger impact. Once included, permanent disability would now dominate the total DALY burden (51\%-55\%) or $80 \%-82 \%$ of total morbidity impact, followed by YLL from mortality (32\%-36\%), and Long COVID (9-10\%). In contrast, when compared to actual COVID-19 cases recorded from January 2020 to January 2021 , mortality drove overall burden of disease from COVID-19 in the first year of the pandemic in Australia, causing some $82 \%$ of the base case DALYs lost or $58 \%$ of Total DALY Burden One, followed by the morbidity impact of permanent disability (29\%). Our results for 2020 are very similar to those recently obtained by the Australian Institute of Health and Welfare (AlHW) [33].

Table 1: Estimated DALY burden for Doherty model scenarios, Base Case (no permanent disability)

\begin{tabular}{|c|c|c|c|c|c|c|c|c|c|c|}
\hline \multicolumn{10}{|l|}{ DALY Burden Estimates - Base Case } & Share of DALY loss for each health state \\
\hline Doherty Scenario & $2 \mathrm{C}$ & $2 \mathrm{D}$ & $3 B$ & $3 C$ & 2020 Actual & $2 \mathrm{C}$ & $2 \mathrm{D}$ & $3 B$ & $3 C$ & 2020 Actual \\
\hline Deaths (no) & 1,524 & 948 & 6,402 & 6,719 & 909 & & & & & \\
\hline COVID cases (no) & 246,399 & 156,799 & 914,357 & 968,154 & 28,696 & & & & & \\
\hline $\begin{array}{l}\text { Mortality } \\
\text { (YLL) }\end{array}$ & 15,912 & 9,898 & 66,844 & 70,154 & 7,263 & $72.6 \%$ & $72.3 \%$ & $74.3 \%$ & $73.8 \%$ & $82.0 \%$ \\
\hline Non-fatal YLD: & & & & & & & & & & \\
\hline Acute & 558 & 353 & 2,069 & 2,213 & 69 & $2.5 \%$ & $2.6 \%$ & $2.3 \%$ & $2.3 \%$ & $0.8 \%$ \\
\hline Long COVID (ONS) & 4,665 & 2,981 & 17,467 & 18,482 & 565 & $21.3 \%$ & $21.8 \%$ & $19.4 \%$ & $19.4 \%$ & $6.4 \%$ \\
\hline PICS & 779 & 462 & 3,578 & 4,198 & 958 & $3.6 \%$ & $3.4 \%$ & $4.0 \%$ & $4.4 \%$ & $10.8 \%$ \\
\hline Permanent & $\mathrm{n} / \mathrm{a}$ & $\mathrm{n} / \mathrm{a}$ & $\mathrm{n} / \mathrm{a}$ & $\mathrm{n} / \mathrm{a}$ & $\mathrm{n} / \mathrm{a}$ & $\mathrm{n} / \mathrm{a}$ & $\mathrm{n} / \mathrm{a}$ & $\mathrm{n} / \mathrm{a}$ & $\mathrm{n} / \mathrm{a}$ & $\mathrm{n} / \mathrm{a}$ \\
\hline Total non-fatal YLD & 6,003 & 3,797 & 23,114 & 24,843 & 1,592 & $27.4 \%$ & $27.7 \%$ & $25.7 \%$ & $26.2 \%$ & $18.0 \%$ \\
\hline Total DALYs (fatal and non-fatal) & 21,915 & 13,695 & 89,958 & 94,998 & 8,855 & $100 \%$ & $100 \%$ & $100 \%$ & $100 \%$ & $100 \%$ \\
\hline
\end{tabular}

Notes: no=number, YLL= years of life lost, YLD= years lived with disability, DALYS= disability adjusted life years, Long COVID ONS= result using ONS data points, PICS= post-Intensive care syndrome and Permanent= Permanent functional impairment, Base case= excluded the burden of permanent disability, results are referring to the combined mean burden of both vaccinated and unvaccinated individuals 
Table 2

Estimated DALY burden for Doherty model scenarios, Total Burden One (permanent disability including diabetes)

DALY Burden Estimates - Total Burden One

Estimated DALY loss for each health state

\begin{tabular}{|c|c|c|c|c|c|c|c|c|c|c|}
\hline Doherty Scenario & $2 \mathrm{C}$ & $2 \mathrm{D}$ & 3B & $3 C$ & 2020 Actual & $2 \mathrm{C}$ & $2 \mathrm{D}$ & $3 B$ & $3 C$ & 2020 Actual \\
\hline Deaths (no) & 1,524 & 948 & 6,402 & 6,719 & 909 & & & & & \\
\hline COVID cases (no) & 246,399 & 156,799 & 914,357 & 968,154 & 28,696 & & & & & \\
\hline Mortality & 15,912 & 9,898 & 66,844 & 70,154 & 7,263 & $32.4 \%$ & $32.2 \%$ & $36.1 \%$ & $35.5 \%$ & $58.3 \%$ \\
\hline
\end{tabular}

(YLL)

Non-fatal YLD:

\begin{tabular}{|c|c|c|c|c|c|c|c|c|c|c|}
\hline Acute & 558 & 353 & 2,069 & 2,213 & 69 & $1.1 \%$ & $1.2 \%$ & $1.1 \%$ & $1.1 \%$ & $0.5 \%$ \\
\hline Long COVID (ONS) & 4,665 & 2,981 & 17,467 & 18,432 & 565 & $9.5 \%$ & $9.7 \%$ & $9.4 \%$ & $9.3 \%$ & $4.5 \%$ \\
\hline PICS & 779 & 462 & 3,578 & 4,198 & 958 & $1.6 \%$ & $1.5 \%$ & $1.9 \%$ & $2.1 \%$ & $7.7 \%$ \\
\hline Permanent & 27,131 & 17,009 & 95,215 & 102,537 & 3,611 & $55.3 \%$ & $55.4 \%$ & $51.4 \%$ & $51.9 \%$ & $29.0 \%$ \\
\hline Total non-fatal YLD & 33,134 & 20,807 & 118,329 & 127,380 & 5,203 & $67.6 \%$ & $67.8 \%$ & $63.9 \%$ & $64.5 \%$ & $41.7 \%$ \\
\hline Total DALYs (fatal and non-fatal) & 49,046 & 30,705 & 185,174 & 197,534 & 12,467 & $100.0 \%$ & $100 \%$ & $100 \%$ & $100 \%$ & $100 \%$ \\
\hline
\end{tabular}

Notes: $n o=n u m b e r, Y L L=$ years of life lost,$Y L D=$ years lived with disability, DALYS = disability adjusted life years, Long COVID ONS= result using ONS data points, PICS= post-Intensive care syndrome and Permanent= Permanent functional impairment, Total burden one= overall burden including all the permanent disability, results are referring to the combined mean burden of both vaccinated and unvaccinated individuals

Table 3

Estimated DALY burden for Doherty model scenarios, Total Burden Two (permanent disability excluding diabetes)

\section{DALY Burden Estimates - Total Burden Two}

Estimated DALY loss for each health state

Share of DALY loss for each health state

\begin{tabular}{|c|c|c|c|c|c|c|c|c|c|c|}
\hline Doherty Scenario & $2 \mathrm{C}$ & $2 \mathrm{D}$ & $3 B$ & $3 C$ & 2020 Actual & $2 \mathrm{C}$ & $2 \mathrm{D}$ & $3 B$ & $3 C$ & 2020 Actual \\
\hline Deaths (no) & 1,524 & 948 & 6,402 & 6,719 & 909 & & & & & \\
\hline COVID cases (no) & 246,399 & 156,799 & 914,357 & 968,154 & 28,696 & & & & & \\
\hline Mortality & 15,912 & 9,898 & 66,844 & 70,154 & 7,263 & $37.0 \%$ & $36.7 \%$ & $40.8 \%$ & $40.2 \%$ & $59.3 \%$ \\
\hline
\end{tabular}

$(\mathrm{YLL})$

Non-fatal YLD:

\begin{tabular}{|c|c|c|c|c|c|c|c|c|c|c|}
\hline Acute & 558 & 353 & 2,069 & 2,213 & 69 & $1.3 \%$ & $1.3 \%$ & $1.3 \%$ & $1.3 \%$ & $0.6 \%$ \\
\hline Long COVID (ONS) & 4,665 & 2,981 & 17,467 & 18,432 & 565 & $10.8 \%$ & $11.1 \%$ & $10.7 \%$ & $10.6 \%$ & $4.6 \%$ \\
\hline PICS & 779 & 462 & 3,578 & 4,198 & 958 & $1.8 \%$ & $1.7 \%$ & $2.2 \%$ & $2.4 \%$ & $7.8 \%$ \\
\hline Permanent & 21,113 & 13,263 & 73,804 & 79,345 & 3,401 & $49.1 \%$ & $49.2 \%$ & $45.1 \%$ & $45.5 \%$ & $27.7 \%$ \\
\hline Total non-fatal YLD & 27,116 & 17,060 & 96,918 & 104,188 & 4,992 & $63.0 \%$ & $63.3 \%$ & $59.2 \%$ & $59.8 \%$ & $40.7 \%$ \\
\hline Total DALYs (fatal and non-fatal) & 43,028 & 26,958 & 163,763 & 174,343 & 12,256 & $100 \%$ & $100 \%$ & $100 \%$ & $100 \%$ & $100 \%$ \\
\hline
\end{tabular}

Notes: $n o=n u m b e r, Y L L=$ years of life lost, YLD= years lived with disability, DALYS = disability adjusted life years, Long COVID ONS $=$ result using ONS data points, PICS= post-Intensive care syndrome and Permanent= Permanent functional impairment, Total burden two= overall burden including all the permanent disability excluding diabetes, results are referring to the combined mean burden of both vaccinated and unvaccinated individuals

Compared with all scenarios included in this analysis, Doherty Scenario 3C generated the highest morbidity and mortality impact followed by Scenario 3B with base case DALY burden of 94,998 (95\% Cl: 88,630-102,559) and 89,958 (95\% Cl:83,925-97,088) respectively. Including the potential impact of permanent functional impairment, the total DALY Burden One was 197,534 (95\%Cl: 189,182-206,262) for Scenario 3C and 185,174 (95\%Cl: 177,383-193,402) for Scenario 3B. Doherty Scenarios 2C and 2D generate significantly lower DALY burden. The base case DALY burden for Scenarios 2C and 2D were 21,915 (95\%Cl:20,278$23,852)$ and $13,695(95 \% \mathrm{Cl}: 12,646-14,921)$ respectively. Total DALY Burden One including the potential burden of permanent function impairment was estimated as $49,046(95 \% \mathrm{Cl}: 47,006-51,193)$ for $2 \mathrm{C}$ and $30,705(95 \% \mathrm{Cl}: 29,409-32,081)$ for $2 \mathrm{D}$. In all these scenarios, total DALY burden is significantly higher in unvaccinated persons than in those vaccinated. (Additional file 4 Table 9).

\section{Sensitivity analysis}

Page 6/12 
As expected, the burden for Long COVID decreased when the NSW estimate was used for Long COVID prevalence rather than ONS (Figure 2). Using the NSWbased prevalence estimates generates a lower estimate of YLD due to Long COVID than when the ONS-based estimate is used (Figure 2 and Additional file 4 Table 9).

Figures 3 and 4 show the potential number of Long COVID cases under Doherty Scenarios 2C-2D, and 3B-3C respectively, using the ONS and NSW Long COVID data points. Scenario 2C and 2D (Figure 3) represent outcomes where Australia has achieved a 70\% vaccination coverage target observing a high seeding infection rate with partial TTIQ. In Scenario 2C, approximately 60,000 (ONS result) or 77,000 (NSW result) Long COVID cases are initially expected from the 244,875 modelled symptomatic COVID-19 infection assuming "low" PHSM. In contrast, a much lower number of Long COVID cases, at approximately 38,000 (ONS result) or 49,000, (NSW result) was estimated for Scenario 2D generated from the 155,851 modelled symptomatic COVID-19 cases assuming medium/low PHSM. For a given vaccine coverage threshold of $80 \%$ and assuming only "baseline" PHSM and partial TTIQ (Figure 4- Scenarios 3B and 3C), both for medium and high seeding infection rate, more than 200,000 (ONS) or >280,000 initial Long COVID cases were estimated from the Doherty model's 908,000 to 961,000 COVID-19 cases. Both figures show how Long COVID case numbers reduce over time as patients recover. Notably, when NSW estimates were used in each scenario, higher Long COVID cases are initially observed, but Long COVID case numbers then decline quite rapidly; when ONS estimates were used, initial Long COVID case numbers are lower, but decline much more slowly.

\section{Discussion}

To the best of our knowledge, this is the first study to illustrate the potential post-acute DALY losses arising from the hypothetical scenarios used by the Doherty Institute[6] to inform Australia's roadmap to reopening its international borders. Based on the Doherty modelling, our study found that even with $80 \%$ full dose vaccination coverage, up to 230,000 initial Long COVID cases might be expected if Australia reopens at a medium or high seeding infection rate (partial TTIQ) with only baseline PHSMs. However, if the Australian vaccination target is at least $70 \%$ and low or medium PHSM are implemented, Long COVID cases will be much lower, even if there is a high seeding infection (partial TTIQ) rate upon reopening.

The Doherty modelling accounts for the impacts of vaccination (and improved treatments) in reducing mortality from COVID-19. Our results show how this is reflected in a declining share of disease burden due to mortality compared with that seen in 2020; and shows how post-acute morbidity will drive an increasing share of COVID-19 disease burden in future. Our study found that Long COVID was responsible for a large proportion of total YLDs (74\%-79\%) in our base case DALY burden and accounted for $19-22 \%$ of the total DALYs lost across scenarios. A key advance in our study was to consider the possible impact of the potential burden of permanent impairment due to COVID-19, which contributed up to $80 \%-82 \%$ of the total YLD or $51 \%-55 \%$ of potential total DALY losses in our model. We found no other studies that have yet estimated the burden of PICS and potential burden of permanent disability in terms of DALYs.

Our results emphasise the continuing importance of high levels of COVID-19 vaccination, not only to reduce mortality and severe acute illness, but also to reduce the burden of Long COVID. At higher levels of vaccine coverage, the relative burden of longer-term morbidity increases, while that of mortality and acute illness decreases; healthcare system responses will increasingly need to deal with a larger post-acute burden of disease. Our results further emphasise a significant finding of the Doherty modelling - namely that, even with high vaccine coverage, specific choices of public health and social distancing measures still have significant impacts on case numbers. Overall COVID burden (acute and post-acute) is significantly higher under "baseline" (i.e. minimal) PHSMs than under low to medium settings. Data on the likely impacts on longer term disease burden therefore must also inform public health decision-making.

Our results indicate that Australian health systems need to prepare to care for many thousands of patients with Long COVID or PICS over coming months. As discussed elsewhere[7], this requires not only specialised Long COVID clinics but, just as importantly, good primary care support and care coordination in the community. Effective surveillance systems need to be established now to identify patients who may go on to develop longer-term sequelae and impairments in the months and years ahead.

We have used the Doherty modelling[6] as our starting point for COVID-19 caseload; as a result, our model therefore shares any limitations inherent to the original Doherty model, including its time horizon of 180 days for new infections. We note that asymptomatic cases were not reported in their report and therefore were excluded in this analysis. This might lead to an underestimate in our DALY estimates if post-COVID sequelae in fact occur in some initially asymptomatic cases. As with similar simulation models, our model used many assumptions. The disability weight for PICS was assumed to be equivalent to QALY utility score of ICU COVID-19 survivors mapped to a similar illness with the same utility score and disability weights. The recovery duration of Long COVID is still unknown and therefore extrapolation of data was necessary. At the time of writing, $81 \%$ of people aged 16 and over had received two doses of vaccine, and Australia looks likely to achieve $90 \%$ coverage levels within weeks; higher vaccination rates will likely further reduce mortality and case numbers, but were not included in the Doherty Institute modelling on which we have based our estimates.

Knowledge of post-COVID-19 sequalae is still evolving and there are inevitable uncertainties around the estimates used in the model. Only the incidence of the most cited permanent disabilities from COVID-19 were included to illustrate the possible additional morbidity from the impact of permanent functional impairment. International literature was used to inform the scale of permanent disability due to scarcity of Australian data.

The precise proportion of COVID-19 survivors who will experience Long COVID is still unknown and different studies reported a wide range of point estimates [7]. UK data[19] from the largest available study were used, but we employed the lower NSW population-based estimate[20] for sensitivity analysis.

To account for the unavoidable uncertainty in the total DALY estimation, we undertook uncertainty analysis. Key inputs such as symptomatic recovery duration was tested using the COVID-19 2020 cases and only a small variation was observed to total DALYs.

\section{Conclusions}


Our results emphasise that ongoing decisions on pandemic control measures and re-opening strategies need to incorporate more than simply numbers of expected infections and deaths. The burden of post-acute COVID-19 was already non-negligible during 2020. However, it becomes relatively more important as deaths fall with improved vaccination coverage. In particular, we have demonstrated that even low rates of incidence of COVID-related permanent illness or disability could still lead to a very significant future burden of disease. Investing now in effective surveillance systems to understand the real incidence and burden of permanent illness or impairment after COVID is therefore essential. Even more urgently, health services must act rapidly to meet the emerging needs of patients with Long COVID, PICS and longer-term illness, not only in the present Victorian and New South Wales outbreaks, but also for the new cases likely to accompany the progressive re-opening of Australia as envisaged in the National Plan [6].

\section{List Of Abbreviations}

AlHW: Australian Institute of Health and Welfare; COVID-19: Coronavirus Disease 2019; DALYs: disability adjusted life years; ICU: intensive care unit; NSW: New South Wales; ONS: Office of National Statistics; OR: odds ratio; PHSM: public health and social measures; PICS: post-intensive care syndrome; UK: United Kingdom; TTIQ: efficacy of test, trace, isolate, quarantine; YLD: years lived with disability; YLL: years lost due to premature mortality

\section{Declarations}

Ethics approval and consent to participate: Not applicable

Consent for publication: Not applicable

Availability of data and materials: All data generated or analysed during this study are included in this published article and provided in Additional files $1,2,3$, and 4.

Competing interests: Authors declare no competing interests

Funding: None received

Author Contributions: All authors contributed equally to the writing and reviewing of the article. MA, LL, MH were responsible in the conceptualisation, modelling and formal analysis of the data. MA, SWAD and DN were responsible in data curation. All authors read and approved the final manuscript.

\section{Acknowledgements}

The authors would like to acknowledge Jan Faller and Jo Perez for their comments and suggestions on previous version of this manuscript.

\section{References}

1. Duckett S, Wood D, Coates B, Mackey W, Crowley T, Stobart A. Race to 80: our best shot at living with COVID: Grattan Institute; 2021; Cited 2021 October 01. Available from: https://grattan.edu.au/wp-content/uploads/2021/07/Race-to-80-our-best-shot-at-living-with-COVID-Grattan-Report.pdf.

2. Kompas T, Grafton RQ, Che TN, Chu L, Camac J. Health and economic costs of early and delayed suppression and the unmitigated spread of COVID-19: The case of Australia. PloS one. 2021;16(6):e0252400.

3. Duckett S, Stobart A. Australia's COVID-19 response: the story so far: Grattan Institute; 2020; Cited 2021 November 08. Available from: https://grattan.edu.au/news/australias-covid-19-response-the-story-so-far/.

4. Department of Health. Government response to the COVID-19 outbreak: Department of Health; 2021; Cited 2021 November 08 . Available from: https://www.health.gov.au/news/health-alerts/novel-coronavirus-2019-ncov-health-alert/government-response-to-the-covid-19-outbreak.

5. Department of the Prime Minister and Cabinet. National Plan to transition Australia's National COVID-19 Response (July 2021) Australia2021; Cited 2021 October 01. Available from: https://www.pmc.gov.au/national-plan-transition-australias-national-covid-response.

6. Doherty Institute. Doherty Modelling Interim report to National Cabinet 17th September 2021 Australia: Doherty Institute; $2021 ;$ Cited 2021 September 20. Available from:

https://www.doherty.edu.au/uploads/content_doc/DOHERTY_MODELLING_INTERIM_REPORT_TO_NATIONAL_CABINET_17TH_SEPTEMBER_2021.pdf.

7. Hensher M, Angeles MR, de Graaf B, Campbell J, Athan E, Haddock R. Deeble Institute Issues Brief No 40. Managing the long term health consequences of COVID-19 in Australia Canberra, Australia: Australian Healthcare and Hospitals Association; 2021; Cited 2021 August 01. Available from:

https://ahha.asn.au/news/long-term-health-consequences-covid-19-can-value-based-health-care-provide-way-forward.

8. Ayoubkhani D, Khunti K, Nafilyan V, Maddox T, Humberstone B, Diamond I, et al. Post-covid syndrome in individuals admitted to hospital with covid-19: retrospective cohort study. BMJ. 2021;372:n693.

9. Taquet M, Geddes JR, Husain M, Luciano S, Harrison PJ. 6-month neurological and psychiatric outcomes in 236379 survivors of COVID-19: a retrospective cohort study using electronic health records. The Lancet Psychiatry. 2021;8(5):416-27.

10. European Burden of Disease Network. Burden of disease of COVID-19: Protocol for country studies 2020; Cited 2021 August 01 Available from: https://www.burden-eu.net/docs/covid19-bod-protocol.pdf.

11. Wyper G, Assunção R, Colzani E, Grant I, Haagsma JA, Lagerweij G, et al. Burden of disease methods: a guide to calculate COVID-19 disability-adjusted life years. International Journal of Public Health. 2021;66:4. 
12. Needham DM, Davidson J, Cohen $\mathrm{H}$, Hopkins RO, Weinert $\mathrm{C}$, Wunsch $\mathrm{H}$, et al. Improving long-term outcomes after discharge from intensive care unit: report from a stakeholders' conference. Crit Care Med. 2012;40(2):502-9.

13. Australian Bureau of Statistics. Table 1.9 Life Tables, Australia, 2017-2019 Canberra: ABS; 2020; Cited 2021 June 23 Available from: https://www.abs.gov.au/statistics/people/population/life-tables/latest-release\#data-download.

14. World Health Organization. The Global Burden of Disease concept The Global Burden of Disease concept; Cited 2021 August 01. 27-40]. Available from: https://www.who.int/quantifying_ehimpacts/publications/en/9241546204chap3.pdf.

15. Global Burden of Disease Collaborative Network. Global Burden of Disease Study 2019 (GBD 2019) Results Seattle, United States: Institute for Health Metrics and Evaluation (IHME); 2020; Cited 2021 August 01 Available from: http://ghdx.healthdata.org/gbd-results-tool.

16. Doherty Institute. Modelling definitions and Frequently Asked Questions 2021; Cited 2021 October 01. Available from: https://www.doherty.edu.au/newsevents/news/modelling-definitions-and-frequently-asked-questions.

17. Byambasuren O, Cardona M, Bell K, Clark J, McLaws M-L, Glasziou P. Estimating the extent of asymptomatic COVID-19 and its potential for community transmission: systematic review and meta-analysis. Official Journal of the Association of Medical Microbiology and Infectious Disease Canada. 2020;5(4):223-34.

18. Cuschieri S, Calleja N, Devleesschauwer B, Wyper GMA. Estimating the direct Covid-19 disability-adjusted life years impact on the Malta population for the first full year. BMC Public Health. 2021;21(1):1827.

19. Office for National Statistics. Prevalence of ongoing symptoms following coronavirus (COVID-19) infection in the UK: 1 April 2021: Estimates of the prevalence of self-reported "long COVID", and the duration of ongoing symptoms following confirmed coronavirus infection, using UK Coronavirus (COVID19) Infection Survey data to 6 March 2021. UK: ONS; 2021; Cited 2021 September 01. Available from:

https://www.ons.gov.uk/peoplepopulationandcommunity/healthandsocialcare/conditionsanddiseases/bulletins/prevalenceofongoingsymptomsfollowin!

20. Liu B, Jayasundara D, Pye V, Dobbins T, Dore GJ, Matthews G, et al. Whole of population-based cohort study of recovery time from COVID-19 in New South Wales Australia. The Lancet Regional Health-Western Pacific. 2021;12:100193.

21. Martin CJ, McDonald S, Luteijn M, Robertson J, Letton W. A model framework for projecting the prevalence and impact of Long-COVID in the UK: medRxiv 2021.05.18.21252341 [Preprint]; 2021; Cited 2021 August 01. Available from: https://doi.org/10.1101/2021.05.18.21252341

22. Antonelli M, Penfold RS, Merino J, Sudre CH, Molteni E, Berry S, et al. Risk factors and disease profile of post-vaccination SARS-CoV-2 infection in UK users of the COVID Symptom Study app: a prospective, community-based, nested, case-control study. The Lancet Infectious Diseases.

23. Zhang J, Yu KF. What's the Relative Risk?A Method of Correcting the Odds Ratio in Cohort Studies of Common Outcomes. JAMA. 1998;280(19):1690-1.

24. Drucker DJ. Diabetes, obesity, metabolism and SARS-CoV-2 infection: The end of the beginning. Cell metabolism. 2021.

25. Lim S, Bae JH, Kwon H-S, Nauck MA. COVID-19 and diabetes mellitus: from pathophysiology to clinical management. Nat Rev Endocrinol. 2021;17(1):1130.

26. Rousseau A-F, Minguet P, Colson C, Kellens I, Chaabane S, Delanaye P, et al. Post-intensive care syndrome after a critical COVID-19: cohort study from a Belgian follow-up clinic. Annals of intensive care. 2021;11(1):1-9.

27. McCue C, Cowan R, Quasim T, Puxty K, McPeake J. Long term outcomes of critically ill COVID-19 pneumonia patients: early learning. Intensive Care Med. 2021;47(2):240-1.

28. Byrnes J, Ball J, Gao L, Kai Chan Y, Kularatna S, Stewart S, et al. Within trial cost-utility analysis of disease management program for patients hospitalized with atrial fibrillation: results from the SAFETY trial. Journal of medical economics. 2019;22(9):945-52.

29. Global Burden of Disease Collaborative Network. Global Burden of Disease Study 2019 (GBD 2019) Disability Weights Seattle, United States: Institute for Health Metrics and Evaluation (IHME); 2020; Cited 2021 August 01 Available from: http://ghdx.healthdata.org/record/ihme-data/gbd-2019-disabilityweights.

30. Cuthbertson BH, Roughton S, Jenkinson D, Maclennan G, Vale L. Quality of life in the five years after intensive care: a cohort study. Crit Care. 2010;14(1):R6-R.

31. Hofhuis JG, Schrijvers AJ, Schermer T, Spronk PE. Health-related quality of life in ICU survivors-10 years later. Scientific Reports. 2021;11(1):1-10.

32. EpiGear International. Ersatz Version 1.35 Brisbane, Australia: EpiGear International; 2017; Cited 2021 August 01. Available from: https://www.epigear.com/index_files/ersatz.html.

33. Australian Institute of Health and Welfare. The first year of COVID-19 in Australia: direct and indirect health effects: Australian Institute of Health and Welfare; 2021; Cited 2021 October 01. Available from: https://www.aihw.gov.au/reports/burden-of-disease/the-first-year-of-covid-19-inaustralia/summary.

\section{Figures}




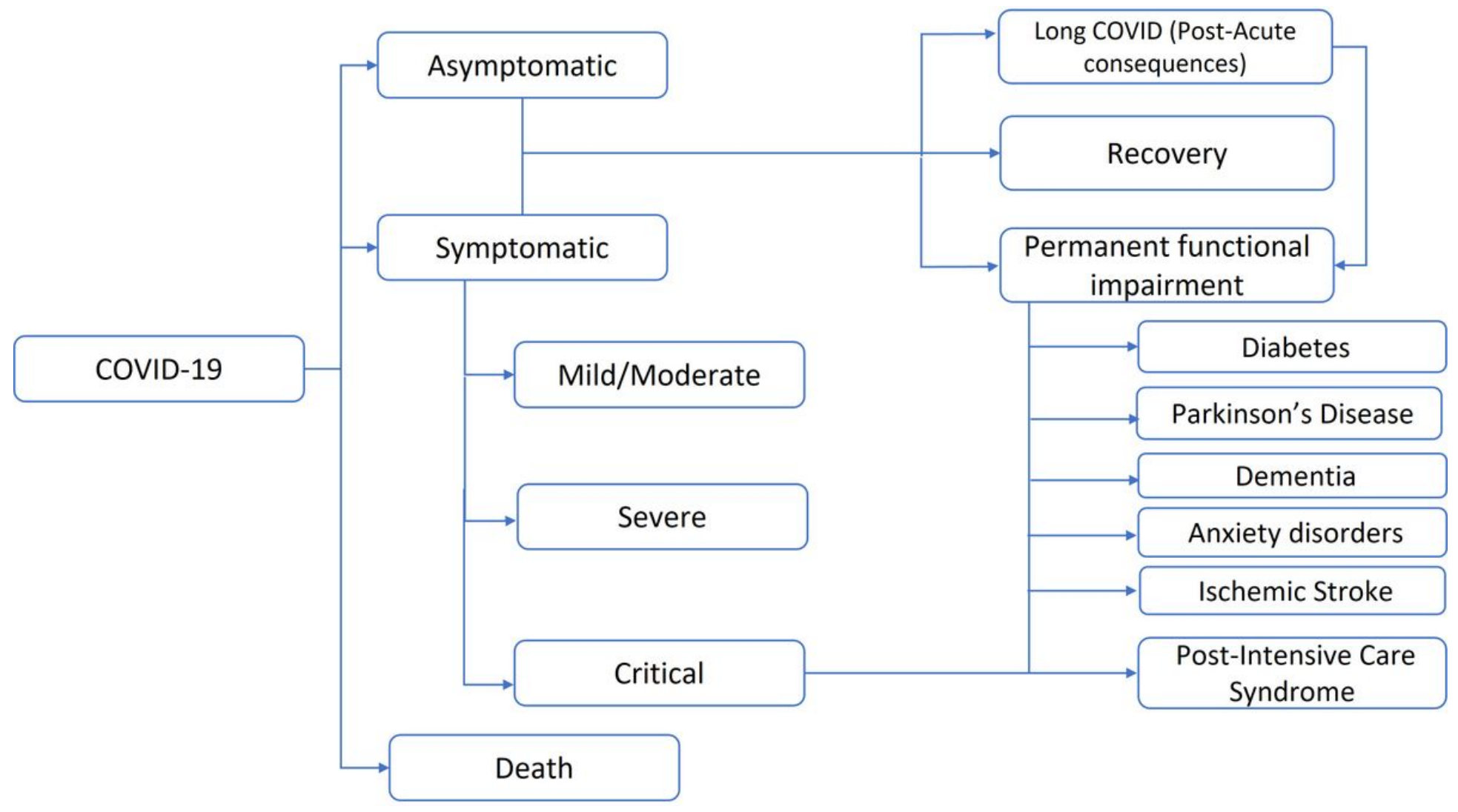

Figure 1

COVID-19 outcome model Source: Adapted from Wyper et al[11] under the Creative Commons Attribution License (CC BY)

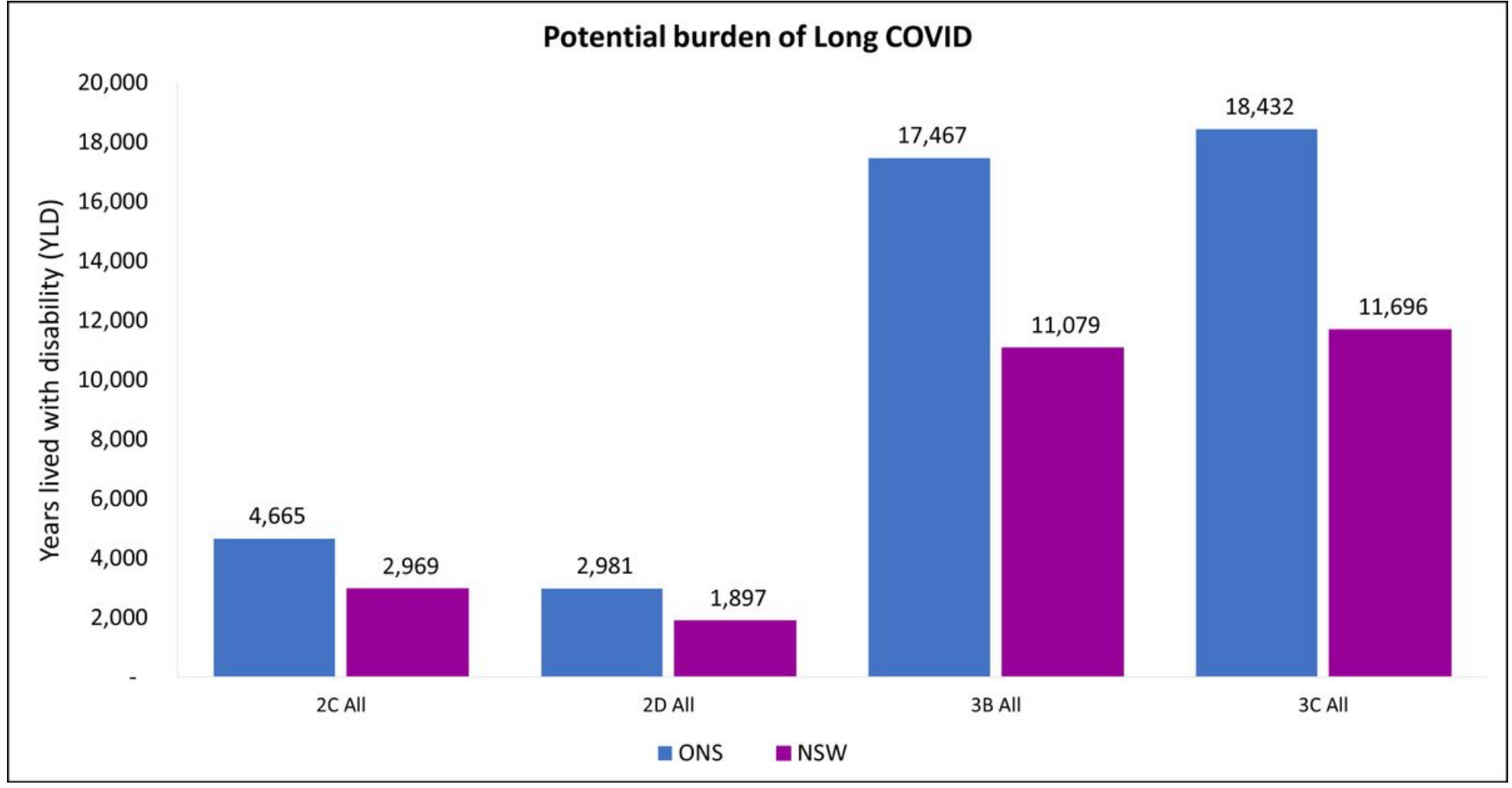

Figure 2

Potential Long COVID burden for all scenarios Notes: ONS= Office of the National Statistic data, NSW=NSW data 


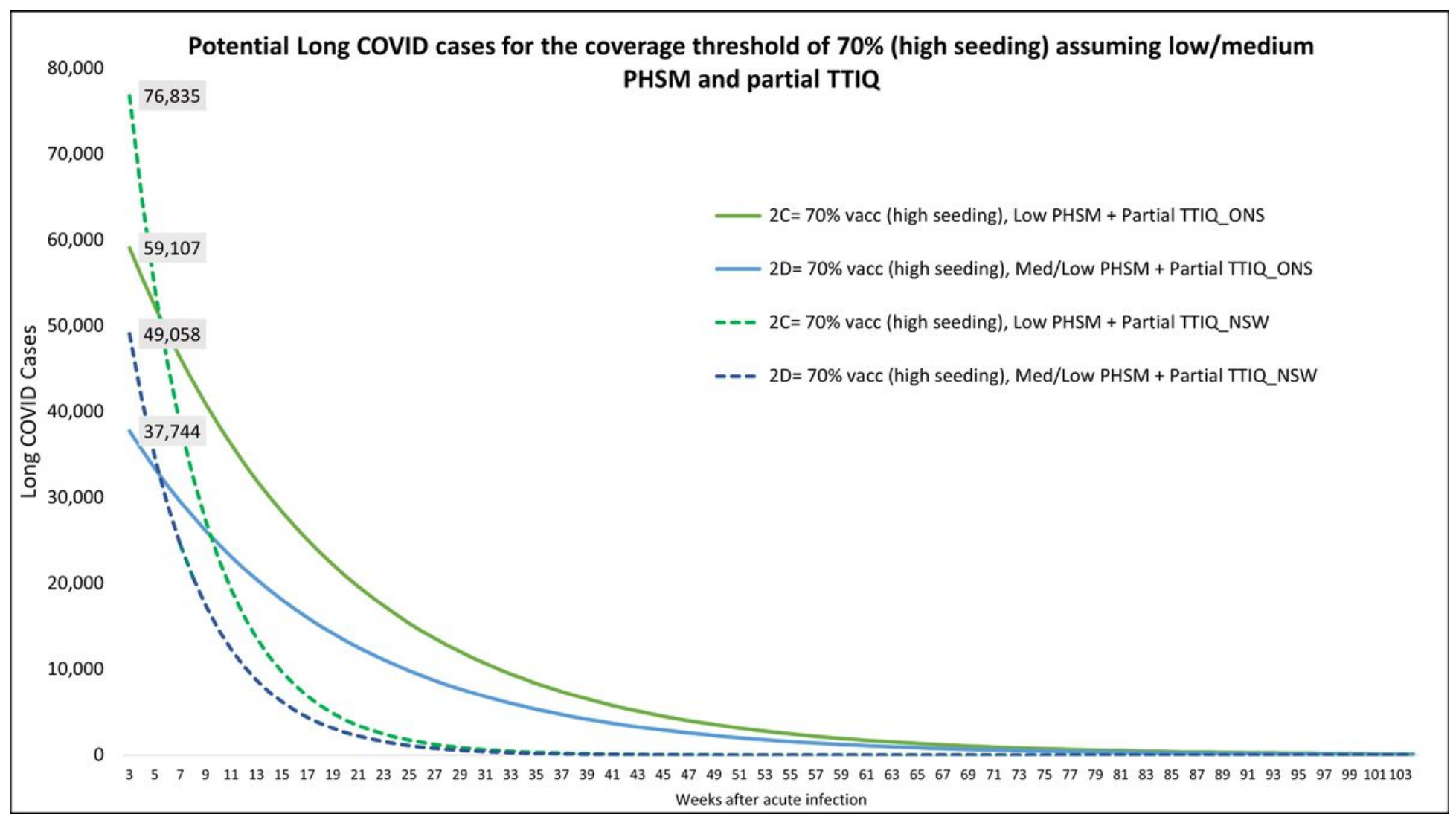

Figure 3

Potential Long COVID cases for Scenarios 2C and 2D Notes: Solid lines presents the results using the ONS data points and Dashed lines presents the results using the NSW datapoints, results noted here excludes the impact of permanent disability and post-intensive care syndrome.

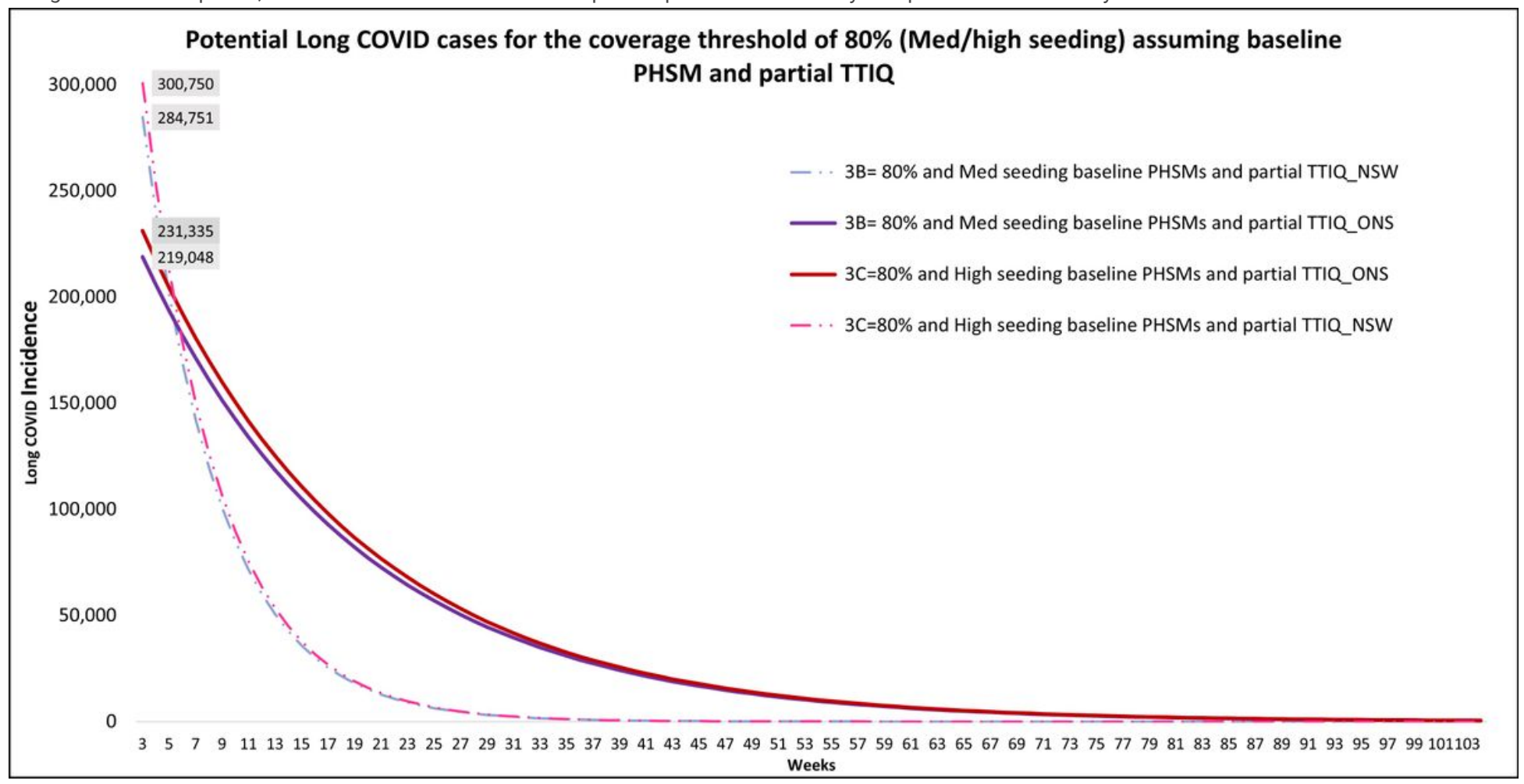

Figure 4

Potential Long COVID cases for Scenarios 3B and 3C Notes: Solid lines presents the results using the ONS data points and Dashed lines presents the results using the NSW datapoints, results noted here excludes the impact of permanent disability and post-intensive care syndrome. 


\section{Supplementary Files}

This is a list of supplementary files associated with this preprint. Click to download.

- Additionalfile1.xlsx

- Additionalfile2.pdf

- Additionalfile3.pdf

- Additionalfile4.pdf 